\title{
NMR study of the high-field magnetic phase of $\mathrm{LiCuVO}_{4}$
}

\author{
N. Büttgen ${ }^{1}$, W. Kraetschmer ${ }^{1}$, L.E. Svistov ${ }^{2}$, L.A. Prozorova ${ }^{2}$, and A. Prokofiev ${ }^{3}$ \\ 1 Center for Electronic Correlations and Magnetism EKM, \\ Experimentalphysik V, Universität Augsburg, D-86135 Augsburg, Germany \\ 2 P.L.Kapitza Institute for Physical Problems RAS, 119334 Moscow, Russia \\ 3 Institut für Festkörperphysik Technische Universität Wien, A-1040 Wien, Austria
}

(Dated: June 14, 2018)

\begin{abstract}
We report on NMR studies of the quasi-1D antiferromagnetic $S=1 / 2$ chain cuprate $\mathrm{LiCuVO}_{4}$, focusing on the high-field spin-modulated phase observed recently in applied magnetic fields $H>$ $H_{\mathrm{c} 2}\left(\mu_{0} H_{\mathrm{c} 2} \approx 7.5 \mathrm{~T}\right)$. The NMR spectra of ${ }^{7} \mathrm{Li}$ and ${ }^{51} \mathrm{~V}$ around the transition from the ordered to the paramagnetic state were measured. It is shown that the spin-modulated magnetic structure forms with ferromagnetic interactions between spins of neighboring chains within the ab-plane at low temperatures $0.6 \mathrm{~K}<T<T_{\mathrm{N}}$. The best fit provides evidence that the mutual orientation between spins of neighboring ab-planes is random. For elevated temperatures $T_{\mathrm{N}}<T \lesssim 15 \mathrm{~K}$, short-range magnetic order occurs at least on the characteristic time scale of the NMR experiment.
\end{abstract}

PACS numbers: 75.50.Ee, 76.60.-k, 75.10.Jm, 75.10.Pq

\section{INTRODUCTION}

The problem of nontrivial ordering in frustrated quantum-spin chains is considered theoretically as a challenging issue $e^{1,2,3,4}$ Recently, the quasi-one-dimensional (1D) antiferromagnetic $S=1 / 2$ chain cuprate $\mathrm{LiCuVO}_{4}$ gained interest as a real material in this context with a low magnetic ordering temperature $T_{\mathrm{N}}$ due to weak inter-

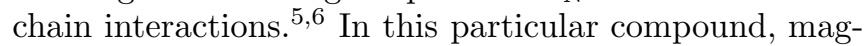
netic frustration due to the intra-chain nearest neighbor (NN) ferromagnetic and the next-nearest neighbor (NNN) antiferromagnetic exchange interactions yields an incommensurate helix structure of the magnetic $\mathrm{Cu}^{2+}$ moments. Additionally, the magnetic structure at $T_{\mathrm{N}}$ is accompanied by ferroelectric order with spontaneous polarization at the same temperature $\frac{7.8}{7.8}$

$\mathrm{LiCuVO}_{4}$ crystallizes in an inverse spinel structure $A B_{2} \mathrm{O}_{4}$ with an orthorhombic distortion induced by a cooperative Jahn-Teller effect of the $\mathrm{Cu}^{2+}$ ions. The elementary cell contains four magnetic ions $\mathrm{Cu}^{2+}(S=$ $1 / 2)$ with the coordinates $(0,0,0),(0,1 / 2,0),(1 / 2,0,1 / 2)$, and $(1 / 2,1 / 2,1 / 2)$ (see Fig. 11). From elastic neutrondiffraction experiments it was established $\underline{9}$ that in the low temperature phase for $T<T_{N}$ and zero applied magnetic field $H=0$ an incommensurate planar spiral spin structure forms which has the propagation wave vector $\mathbf{k}_{\mathbf{i c}}$ directed along the $\mathrm{Cu}^{2+}$ chains $\left(\mathbf{k}_{\mathbf{i c}} \| \mathbf{b}\right.$, see Fig. 1). We parametrized the helix of this spin structure with magnetic moments $\mu$ of the $\mathrm{Cu}^{2+}$ ions utilizing the coordinates $x, y$, and $z$ along the $\mathbf{a}, \mathbf{b}$, and $\mathbf{c}$ directions, respectively (Ref. 10):

$$
\begin{array}{r}
\mu(x, y, z)=\mu_{\mathrm{Cu}} \cdot \mathbf{l}_{\mathbf{1}}(-1)^{2 z / c} \cdot \cos \left(k_{i c} \cdot y+\phi\right)+ \\
\mu_{\mathrm{Cu}} \cdot \mathbf{l}_{\mathbf{2}}(-1)^{2 z / c} \cdot \sin \left(k_{i c} \cdot y+\phi\right),
\end{array}
$$

where $\mathbf{l}_{\mathbf{1}}$ and $\mathbf{l}_{\mathbf{2}}$ are orthogonal unit vectors within the ab-plane. At zero applied magnetic field $H=0$, the absolute value of the propagation wave vector is $k_{\text {ic }}=$

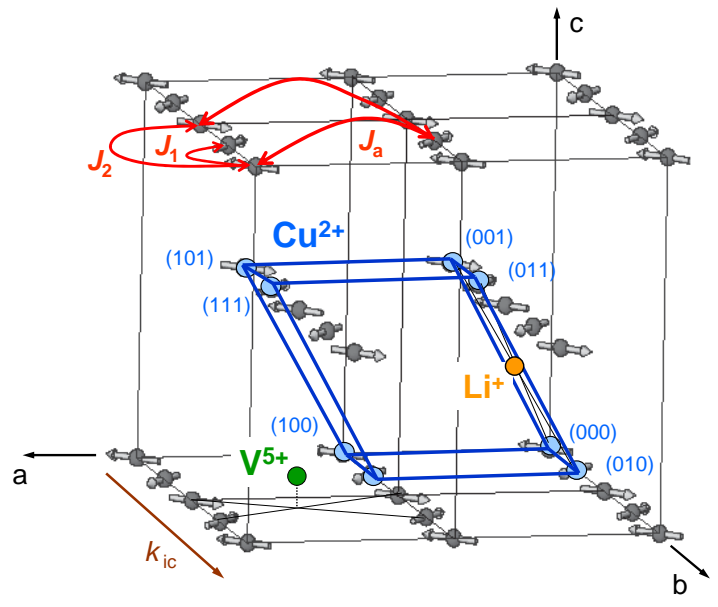

FIG. 1: Scheme of the $\mathrm{Cu}^{2+}$ moments in the crystal structure of $\mathrm{LiCuVO}_{4}$ (Ref. 9). Copper ions are marked by grey circles, where the arrows at each site constitute the helical spin structure at $H=0$ below $T_{\mathrm{N}}$ (Ref. 9). $J_{1}, J_{2}, J_{a}$ (red arrows) denote the main exchange integrals ${ }^{11}$ defining the magnetic structure of $\mathrm{LiCuVO}_{4}$. Additionally, the positions of $\mathrm{Li}$ (orange) and $\mathrm{V}$ (green) ions are exemplarily depicted by one ion each. In favor of clarity, the $\mathrm{V}^{5+}$ ion is shifted into the foreground of the cell.

$(1-0.532) \cdot 2 \pi / b$ and the ordered $\mathrm{Cu}$ moment amounts to $\mu_{\mathrm{Cu}}=0.31 \mu_{B}$ (Refs. 9, 11). The angle $\phi$ in Eq. 1 denotes an arbitrary phase shift.

Inelastic neutron-scattering experiments 11 confirmed that the magnetically ordered structure of $\mathrm{LiCuVO}_{4}$ is quasi-1D with dominating exchange interactions $J_{1,2}$ within the chains. It was shown that the incommensurate structure is due to strong intra-chain NN ferromagnetic and NNN antiferromagnetic exchange interactions with $J_{1}=-18 \mathrm{~K}$ and $J_{2}=49 \mathrm{~K}$, respectively (see Fig. 11). Note that this hierarchy of interactions causes strong magnetic frustration. A three dimensional magnetic order results from ferromagnetic exchange in- 


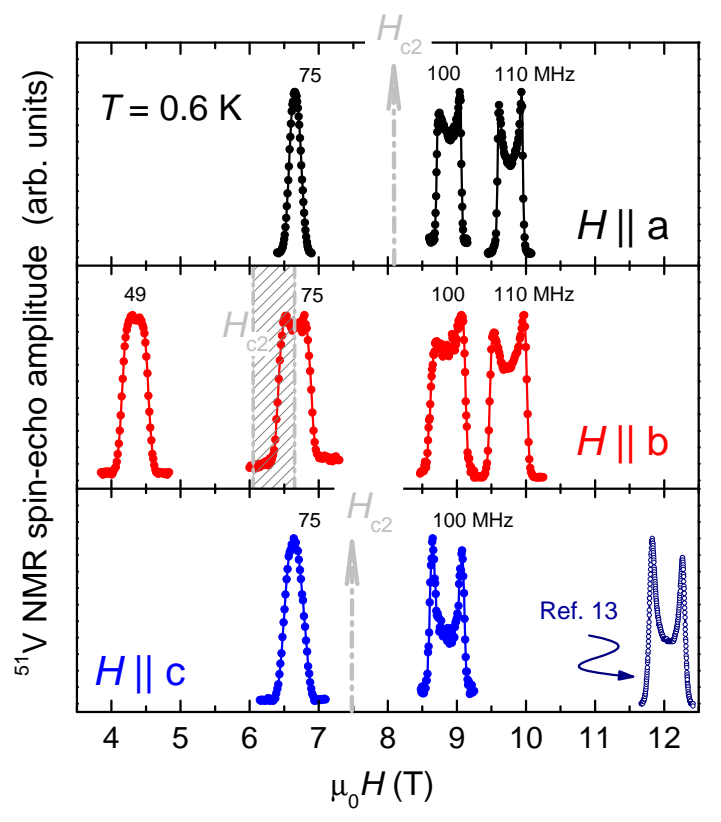

FIG. 2: NMR spectra of ${ }^{51} \mathrm{~V}$ measured at $T=0.6 \mathrm{~K}$ for different frequencies, i.e. different applied magnetic fields $H$. The spectrum obtained in Ref. 13 at $\mathrm{T}=1.64 \mathrm{~K}$ is included.

teractions $J_{a} \approx-4.3 \mathrm{~K}$ between magnetic moments of neighboring chains within an ab-plane (red arrows in Fig. 1), and about five times weaker interactions between magnetic moments of different $\mathbf{a b}$-planes. By application of a static magnetic field $H$, a number of consecutive magnetic phase transitions was observed with increasing field $10,11,12$ The saturation fields were determined to be $\mu_{0} H_{s} \approx 40 \mathrm{~T}$ and $50 \mathrm{~T}$ for the orientations $\mathbf{H} \| \mathbf{c}$ and $\mathbf{H} \| \mathbf{a}$, respectively. In the low-field range $\left(\mu_{0} H<12 \mathrm{~T}\right)$ the magnetically ordered structures were studied by means of ESR and NMR techniques, $\underline{\underline{10}}$ On increasing the field $\mu_{0} H>0$, the first phase transition takes place at $\mu_{0} H_{c 1} \approx 2.5 \mathrm{~T}$ with the direction of $\mathbf{H}$ applied within the $\mathbf{a b}$-plane. It can be explained as a spin-flop reorientation of the spiral spin plane of the magnetic structure where the spin plane is oriented perpendicularly to the applied magnetic field according to $\mathbf{H} \|\left(\mathbf{l}_{\mathbf{1}} \times \mathbf{l}_{\mathbf{2}}\right)$. The transition field $\mu_{0} H_{c 1}$ is determined by the value of the anisotropic exchange and by the anisotropy of the magnetic susceptibility.

A more interesting and unexpected phase transition is observed at higher fields $\mu_{0} H_{c 2} \approx 7.5 \mathrm{~T}$. The observation of this magnetic transition for all three directions $\mathbf{H} \| \mathbf{a}, \mathbf{b}, \mathbf{c}$ reveals an exchange nature of this transition. The NMR spectra observed at $H>H_{c 2}$ can only be explained by the assumption that a spin-modulated structure is realized, where the ordered component of the spin structure is oriented parallel to the applied magnetic field $\mathbf{H} \| \mathbf{l}_{\mathbf{1}}$ and $\mathbf{l}_{\mathbf{2}}=0$. An indirect confirmation of this sequence of magnetic phase transitions suggested in Ref. 10 was obtained by experimental investigation of the dielectric properties of $\mathrm{LiCuVO}_{4} \stackrel{8}{\stackrel{8}{*} \mathrm{~A}}$ closer inspection of

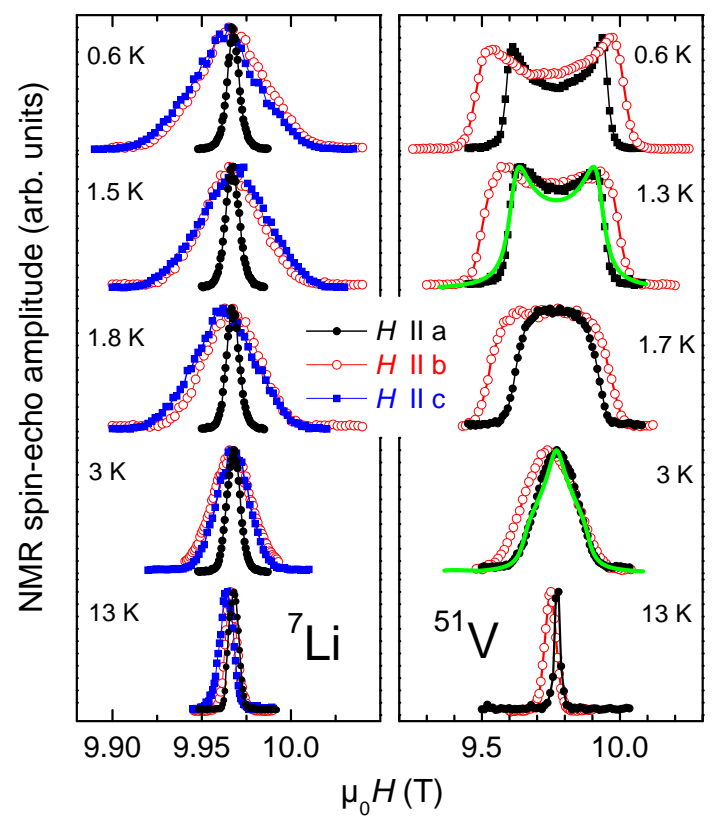

FIG. 3: NMR spectra of ${ }^{7} \mathrm{Li}(165 \mathrm{MHz}$, left column) and ${ }^{51} \mathrm{~V}(110 \mathrm{MHz}$, right column) at different temperatures for $H>H_{c 2}$. Additionally, the ${ }^{51} \mathrm{~V}$ spectra at $T=1.3 \mathrm{~K}$ and $3 \mathrm{~K}$ for $\mathbf{H} \| \mathbf{a}$ are displayed together with simulation curves (green solid lines).

the high-field phase for $H>H_{c 2}$ will be given in this work by means of NMR spectroscopy and different scenarios are discussed for the collinear magnetic structure which is realized for $H>H_{c 2}$.

\section{NMR EXPERIMENT}

The single crystal under investigation is the identical crystal used in Refs. 8 and 10. The NMR experiments were performed with a phase coherent, homemade spectrometer at radio frequencies within the range $45.1<\nu<165 \mathrm{MHz}$ (for details see Ref. 10). The effective local magnetic field acting on nuclei of a nonmagnetic ion is determined by long-range dipolar fields from the surrounding magnetic ions and by so called 'contact' hyperfine fields from NN magnetic ions. The positions of $\mathrm{Li}^{1+}$ and $\mathrm{V}^{5+}$ ions surrounded by the copper ions are shown in figure 1. The $\mathrm{Li}^{1+}$ ions are located between the $\mathbf{a b}$-plane of $\mathrm{Cu}$ ions and the $\mathrm{V}^{5+}$ ions are very close to one single ab-plane of $\mathrm{Cu}$ ions. Therefore, the ${ }^{7} \mathrm{Li}$ NMR spectra are sensitive to the mutual orientation between $\mathrm{Cu}$ spins of adjacent $\mathbf{a b}$-planes. In case of the ${ }^{51} \mathrm{~V}$ $\mathrm{NMR}$ in $\mathrm{LiCuVO}_{4}$, this mutual orientation between the $\mathrm{Cu}$ spins of adjacent $\mathbf{a b}$-planes does not affect the spectral shape, because the effective local magnetic field at the V sites is dominated by contact hyperfine fields from the four $\mathrm{Cu}^{+2}$ moments of the nearest $\mathbf{a b}$-plane.

Figure 2 shows the ${ }^{51} \mathrm{~V}$ NMR spectra at different frequencies, i.e. different applied magnetic fields, for all 
three directions $\mathbf{H} \| \mathbf{a}, \mathbf{b}, \mathbf{c}$. All spectra with resonance fields $H<H_{c 2}$ exhibit one single line, irrespective of the orientation of the applied magnetic field. For fields $H>H_{c 2}$, the NMR spectra have the characteristic double-horn shape which is a fingerprint of modulated magnetic structures. The arrows in Fig. 2 indicate the anisotropy of the transition fields $H_{c 2}$ obtained in Refs. 8, 10 and 12. For $\mathbf{H} \| \mathbf{b}$, the value for $H_{c 2}$ is obtained from NMR data: at magnetic fields $\mu_{0} H \approx 6.7 \mathrm{~T}$, the ${ }^{51} \mathrm{~V}$ NMR spectral line already transforms to the doublehorn pattern which is specific for the high-field magnetic phase (see the middle frame in Fig. 2). From the ${ }^{7} \mathrm{Li}$ NMR spectral shape for $\mathbf{H} \| \mathbf{b}$ it was concluded that $\mu_{0} H_{c 2}>6.05 \mathrm{~T}$ (cf. Ref. 10). Therefore, we find the value of $H_{c 2}$ for the orientation $\mathbf{H} \| \mathbf{b}$ within the range $6.05<\mu_{0} H_{c 2}<6.7 \mathrm{~T}$. This uncertainty is depicted in the middle frame of Fig. 2 by the hatched area. The single line of the ${ }^{51} \mathrm{~V}$ NMR spectra which is obtained for all three orientations for $H_{c 1}<H<H_{c 2}$ shows that the spin rotating axis of the helical magnetic structure is parallel to the applied field $\mathbf{H} \|\left(\mathbf{l}_{\mathbf{1}} \times \mathbf{l}_{\mathbf{2}}\right)$ confirming the observations made in reference 10 .

Figure 3 shows the temperature dependences of the NMR spectra of ${ }^{7} \mathrm{Li}$ and ${ }^{51} \mathrm{~V}$ at irradiation frequencies of $165 \mathrm{MHz}$ and $110 \mathrm{MHz}$, respectively. These frequencies were chosen in order to obtain NMR spectra of both nuclei at the same applied magnetic field around $\mu_{0} H=10 \mathrm{~T}$, exceeding the transition field $H_{c 2}$. The ${ }^{7} \mathrm{Li}$ spectra for all temperatures exhibit a single line whereas the ${ }^{51} \mathrm{~V}$ spectra in the low-temperature range reveal the double-horn pattern. For temperatures $T<1.3 \mathrm{~K}$, the NMR spectral shapes occur to be temperature independent. As it was shown in Ref. 10 such spectral shapes of both nuclei can only be realized for a collinear spinmodulated structure with $\mathbf{H} \| \mathbf{l}_{\mathbf{1}}$ and $\mathbf{l}_{\mathbf{2}}=0$. Figure 4 gives the temperature dependences of the NMR line

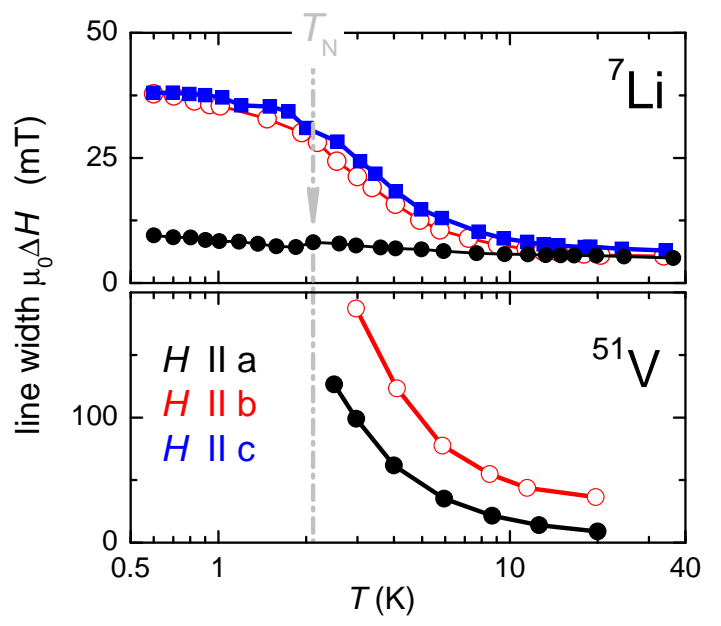

FIG. 4: Temperature dependences of the line widths $\Delta H(T)$ obtained from Gaussian fits to the data in Fig. 3 at $H>H_{\mathrm{c} 2}$. Upper and lower frame for ${ }^{7} \mathrm{Li}$ and ${ }^{51} \mathrm{~V}$ nuclei, respectively. $T_{\mathrm{N}} \approx 2.1 \mathrm{~K}$ is marked by an arrow (see text). width of ${ }^{7} \mathrm{Li}$ and ${ }^{51} \mathrm{~V}$ obtained from Gaussian fits of the data presented in figure 3. In case of the ${ }^{51} \mathrm{~V}$ spectra, such fitting to Gaussian lines was only possible for temperatures $T>2.1 \mathrm{~K}$, because the spectral shape for $T<2.1 \mathrm{~K}$ changes to a broad plateau-like pattern. It is important to note that there is an abrupt $10 \%$ steplike decrease of the ${ }^{7} \mathrm{Li}$ line width $\Delta H(T)$ at around $2 \mathrm{~K}$ for the field orientation $\mathbf{H} \| \mathbf{a}$. Thus, the temperature $T_{\mathrm{N}} \approx 2.1 \mathrm{~K}$ identifies the transition temperature into the magnetically ordered phase in agreement with the phase diagram established in Refs. 8 and 12 . At elevated temperatures $T_{\mathrm{N}}<T<15 \mathrm{~K}$, the line widths $\Delta H(T)$ for both nuclei decrease with increasing temperature. A temperature independent, isotropic line width $\Delta H$ which is characteristic for paramagnetic behavior is only established for $T>15 \mathrm{~K}$, far above $T_{\mathrm{N}}$.

\section{DISCUSSION AND CONCLUSION}

In order to further investigate this high-field phase $H>H_{c 2}$ we will consider two types of magnetic structures which assure such modulated spin components directed along $H$, i.e. a planar spiral spin structure with $\mathbf{H} \perp \mathbf{n}$ (where $\mathbf{n}=\mathbf{l}_{\mathbf{1}} \times \mathbf{l}_{\mathbf{2}}$ ) and a collinear structure with $\mathbf{H} \| \mathbf{l}_{\mathbf{1}}$, respectively.

Moreover, we take into account the hierarchy of the magnetic exchange interactions in $\mathrm{LiCuVO}_{4}$. As the exchange interactions within the $\mathbf{a b}$-plane greatly exceed the inter-plane interactions, we will test on one hand the case with long-range magnetic order in $\mathbf{c}$-direction (3D in the following), and on the other hand the case of a long-range order within the ab-planes but an arbitrary mutual orientation of spins along the $\mathbf{c}-$ direction, i.e. between different $\mathbf{a b}$-planes (2D).

Figure 5 presents the experimental ${ }^{7} \mathrm{Li}$ NMR spectra at $T=0.6 \mathrm{~K}$ (symbols) and the simulated spectra (solid lines) according to the suggested spin structures mentioned above. The simulations result from calculations of the $\mathrm{Cu}^{2+}$ dipolar fields at the probing ${ }^{7} \mathrm{Li}$ nuclear site (cf. Ref. 10). The left (right) column of Fig. 5 shows the simulated ${ }^{7} \mathrm{Li}$ NMR spectra for the planar spiral spin structure (collinear spin-modulated structure), respectively. The 3D long-range magnetic order is taken into account by a constant phase $\phi(x, z)$ in Eq. 11 whereas in case of the 2D long-range magnetic order the phase $\phi$ is only constant with respect to $x$ but arbitrary with respect to the $z$-coordinate $[\phi(x, z)=\operatorname{random}(z)]$. The best agreement between experiment and simulations is obtained for the spin-modulated 2D magnetic structure, i.e. with a random phase relation between spins along the $c$-axis (cf. lower right frame in Fig. 5).

The incommensurate propagation wave vector $\mathbf{k}_{\mathbf{i c}}$ at high applied magnetic fields is experimentally unknown, but the simulations revealed that a variation of $k_{\text {ic }}$ does not change the shape of the calculated NMR spectra. Thus, at low temperatures and high magnetic fields $H>H_{c 2}$ long-range magnetic order in the ab-plane 


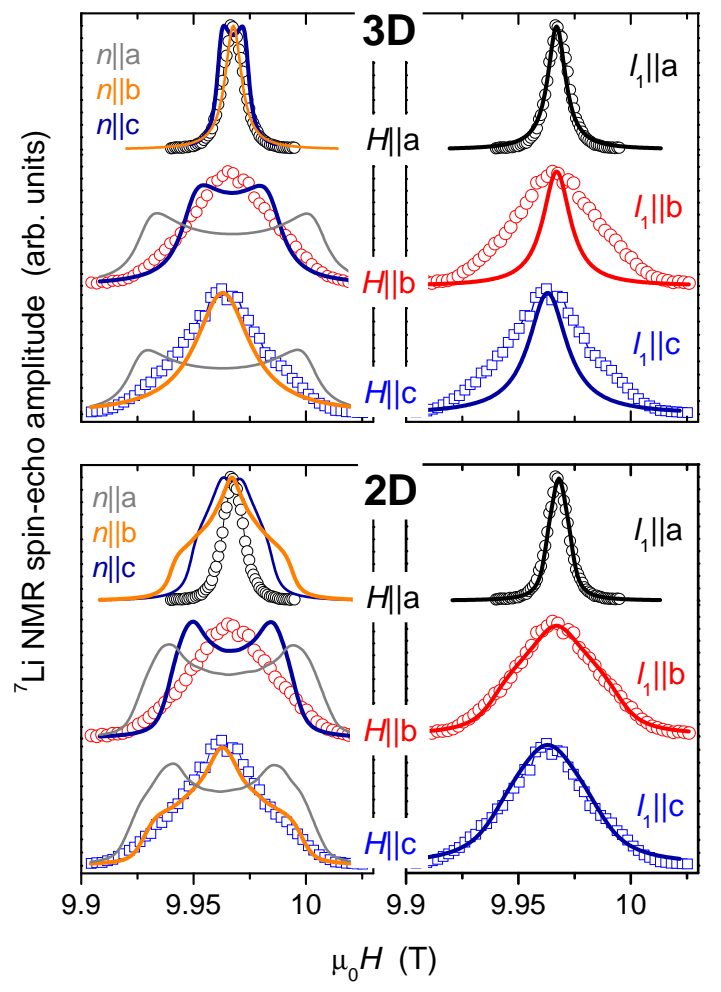

FIG. 5: ${ }^{7} \mathrm{Li}$ NMR spectra at $T=0.6 \mathrm{~K}$ and $165 \mathrm{MHz}$ (symbols). The solid lines represent the simulations (see text): left (right) column shows the simulated spectra for the planar spiral structure with $\mathbf{H} \perp \mathbf{n}$ (collinear spin-modulated structure, $\mathbf{H} \| \mathbf{l}_{\mathbf{1}}$ and $\mathbf{l}_{\mathbf{2}}=0$ ), respectively.

without inter-plane coupling along the $c$-direction is realized in $\mathrm{LiCuVO}_{4}$. This proposed magnetic structure is consistent with the ${ }^{51} \mathrm{~V}$ NMR spectra: the effective magnetic fields on these nuclei are dominated by contact fields of the four neighboring $\mathrm{Cu}$ ions of the nearest $\mathbf{a b}$-plane (see Fig. 1). Therefore, these spectra are not sensitive to magnetic disorder in $c$-direction. This explains the double-horn shape of the ${ }^{51} \mathrm{~V}$ NMR spectra for $H>H_{c 2}$ at very low temperatures $T=0.6 \mathrm{~K}$. In addition, the lack of a spiral spin structure proposed for $H>H_{c 2}$ accounts for the paraelectric behavior according to reference 8 .

At elevated temperatures $T \gtrsim 2 \mathrm{~K}$, the shape of the ${ }^{51} \mathrm{~V}$ NMR spectra transforms from the double-horn shape at low temperatures to a single line pattern which can be fitted by Gaussian lines. In the lower frame of figure 4. the temperature dependence of the line width $\Delta H$ of these Gaussian lines is plotted. It is important to note that broad Gaussian lines can be observed up to approximately $15 \mathrm{~K}$ indicating short-range magnetic ordering at the time scale of the NMR experiment, far above the ordering temperature $T_{\mathrm{N}} \approx 2 \mathrm{~K}$. A lower bound of the time scale is given by the time for spin-echo formation which was achieved in our experiments with pulse separation times $\tau_{D}=35 \mu \mathrm{s}$. From the spin-lattice relaxation time $T_{1}$ of our measurements we estimate an extension of the time scale even to milliseconds.

Most probably this transformation of the ${ }^{51} \mathrm{~V}$ NMR spectral shape (see right column in Fig. 3) indicates the loss of ferromagnetic interactions along the a-direction towards higher temperatures. In order to corroborate this assumption we simulated the ${ }^{51} \mathrm{~V}$ NMR spectra at 1.3 and $3 \mathrm{~K}$ for this scenario. The simulated spectra are included in Fig. 3 (right column, green solid lines): the simulation at $1.3 \mathrm{~K}$ is based on the spin-modulated $2 \mathrm{D}$ magnetic structure with random phase relation $\phi$ along the c-direction mentioned above for the ${ }^{7} \mathrm{Li} \mathrm{NMR}$ spectra. For the simulation at $3 \mathrm{~K}$, the release of ferromagnetic interactions between neighboring chains in the $\mathbf{a}^{-}$ direction towards higher temperatures is taken into account by randomizing the phase relation $\phi$ along the $\mathbf{a}^{-}$ direction as well $[\phi(x, z)=\operatorname{random}(x, z)$ in Eq. 1 .

In conclusion, the magnetic structure of the highfield magnetic phase of the quasi-1D antiferromagnet $\mathrm{LiCuVO}_{4}$ was studied by NMR experiments. We determined that the spin-modulated magnetic structure $(\mathbf{H} \|$ $\mathbf{l}_{\mathbf{1}}$ ) with long-range magnetic order within the $\mathbf{a b}$-plane and a random phase relation between the spins of neighboring ab-planes is realized in $\mathrm{LiCuVO}_{4}$ at $H>H_{\mathrm{c} 2}$ and low temperatures $T<T_{\mathrm{N}}$. The observed NMR spectra can be satisfactorily described by the following structure:

$$
\mu(x, y, z)=\mu_{\mathrm{Cu}} \cdot \mathbf{l} \cdot \cos \left[k_{i c} \cdot y+\phi(z)\right]
$$

where $\mathbf{l}$ is the unit vector parallel to the applied magnetic field $\mathbf{H}$ and the phase $\phi(z)$ between adjacent spins in $\mathbf{c}$-direction is random. Within the temperature range $T_{\mathrm{N}}<T \lesssim 15 \mathrm{~K}$, the NMR spectra can be described if we assume that the phase relation $\phi$ between neighboring spin chains in the ab-plane is random too. The particular phases for both structures below and above $T_{\mathrm{N}}$ for $H>H_{\mathrm{c} 2}$ are time independent at least on the characteristic time scale of the NMR experiment of microseconds.

\section{Acknowledgments}

This work is supported by the Grants 09- 0212341, 100201105-a Russian Foundation for Basic Research, Russian President Program of Scientific Schools, and by the German Research Society (DFG) within the Transregional Collaborative Research Center (TRR 80).
1 A.V. Chubukov, Phys. Rev. B 44, 4693 (1991).

2 A.K. Kolezhuk, Phys. Rev. B 62, R6057, (2000).

3 A.K. Kolezhuk and T. Vekua, Phys. Rev. B 72, 094424
(2005).

4 D.V. Dmitriev and V.Yu. Krivnov, Phys. Rev. B 77, 024401 (2008). 
5 J. Sudan, A. Lüscher, and A.M. Läuchli, Phys. Rev. B 80, 140402(R) (2009).

${ }^{6}$ F. Heidrich-Meisner, I.P. McCulloch, and A.K. Kolezhuk, Phys. Rev. B 80, 144417 (2009).

7 Y. Naito et al., J. Phys. Soc. Jpn. 76, 023708 (2007).

8 F. Schrettle et al., Phys. Rev. B 77, 144101 (2008).

9 B.J. Gibson et al., Physica B 350, e253 (2004).
10 N. Büttgen et al., Phys. Rev. B 76, 014440 (2007).

11 M. Enderle et al., Europhys. Lett. 70, 237 (2005).

12 M.G. Banks et al., J. Phys.: Condens. Matter 19, 145227 (2007)

13 R. Smith et al., Physica B 378-380, 1060 (2006). 